\title{
Estimation Risk Modeling in Optimal Portfolio Selection: An Empirical Study from Emerging Markets
}

\author{
Sarayut Nathaphan ${ }^{1}$ and Pornchai Chunhachinda ${ }^{2}$ \\ ${ }^{1}$ Business Division, Mahidol University International College, Nakhonpathom 73170, Thailand \\ ${ }^{2}$ Thammasat Business School, Thammasat University, Bangkok 10200, Thailand
}

Correspondence should be addressed to Sarayut Nathaphan, sarayut.mark@gmail.com

Received 3 March 2010; Accepted 22 June 2010

Academic Editor: Benjamin Miranda Tabak

Copyright (c) 2010 S. Nathaphan and P. Chunhachinda. This is an open access article distributed under the Creative Commons Attribution License, which permits unrestricted use, distribution, and reproduction in any medium, provided the original work is properly cited.

\begin{abstract}
Efficient portfolio is a portfolio that yields maximum expected return given a level of risk or has a minimum level of risk given a level of expected return. However, the optimal portfolios do not seem to be as efficient as intended. Especially during financial crisis period, optimal portfolio is not an optimal investment as it does not yield maximum return given a specific level of risk, and vice versa. One possible explanation for an unimpressive performance of the seemingly efficient portfolio is incorrectness in parameter estimates called "estimation risk in parameter estimates". Six different estimating strategies are employed to explore ex-postportfolio performance when estimation risk is incorporated. These strategies are traditional Mean-Variance (EV), Adjusted Beta (AB) approach, Resampled Efficient Frontier (REF), Capital Asset Pricing Model (CAPM), Single Index Model (SIM), and Single Index Model incorporating shrinkage Bayesian factor namely, Bayesian Single Index Model (BSIM). Among the six alternative strategies, shrinkage estimators incorporating the single index model outperform other traditional portfolio selection strategies. Allowing for asset mispricing and applying Bayesian shrinkage adjusted factor to each asset's alpha, a single factor namely, excess market return is adequate in alleviating estimation uncertainty.
\end{abstract}

\section{Introduction}

Efficient portfolio is a portfolio that yields maximum expected return given a level of risk or has a minimum level of risk given a level of expected return. Traditional efficient portfolio and its extension incorporating single factor model as suggested by Markowitz [1], Sharpe [2], and Elton, Gruber, Padberg [3], and Michaud [4] had been explored and implemented in active portfolio management. Optimal portfolio or the active portfolio is determined at the tangency of the capital allocation line and the efficient frontier. Portfolio or asset allocation came into play dividing individual wealth investing in three investment choices. The first choice of investment is an active portfolio, the second is the market index portfolio or passive portfolio, and the third is riskless asset or cash. However, performance of an investment strategy recommended by a fund manager, mostly, is not impressive. Especially during the financial crisis period, optimal portfolio is not an optimal investment as intended. One possible explanation for an unimpressive performance of the seemingly efficient portfolio is incorrectness in parameter estimates called "estimation risk in parameter estimates". Two crucial parameters in an efficient portfolio construction are expected return and variancecovariance matrix. Estimation risk in portfolio formation is caused by treating sample estimates as true parameters. This paper aims at taking estimation risk in parameter estimates into account when constructing an efficient frontier using empirical Bayesian shrinkage incorporating single factor (index) model and comparing Bayesian portfolio's performance with other portfolio formation strategies during two financial crisis periods.

Various studies in the past can be divided into three groups. The first group conducted their studies based on historical data ignoring estimation risk. This group includes Markowitz [1], Sharpe [2], Kraus and Litzenberger [5], Kroll, Levy and Markowitz [6], and Chunhachinda et al. $[7,8]$. The second group of studies took estimation risk 
into account by proposing a Bayesian or resample efficient frontier approach using historical data together with Monte Carlo estimation process; for example, Stein [9], Kalymon [10], Barry [11], Klein and Bawa [12], Brown [13], Chen and Brown [14], Jorion [15], Horst et al. [16], Markowtiz and Usmen [17], and Michaud [18]. The third group focused on the asset pricing approach by incorporating a factor model such as the Capital Asset Pricing Model (CAPM) and/or Arbitrage Pricing Theory (APT) in the portfolio selection process, for example, Polson and Tew [19], and Pāstor [20]. The third group uses a factor model to benchmark the performance of a recommended portfolio. Portfolio optimization is performed based on historical data to estimate two crucial parameters of the model, namely, expected return and variance-covariance matrix. Estimation risk due to treating sample estimates as true parameters had been taken into account in optimal portfolio formation via Bayesian Portfolio Optimization process.

Studies regarding the effect of estimation risk on an optimal portfolio have been conducted by a lot of scholars. Barry [11] stated that estimation risk does not change the efficient set but will affect the optimal portfolio. Bawa, Brown, and Klein [21] and Klein and Bawa [12] stated that, when estimation risk is taken into account, assuming that security returns are generated by a stationary multivariate normal distribution for which the investor has a diffuse prior, the effects of estimation risk on the selection of an optimal portfolio from a set of risky assets cause the location, but not the composition, of the efficient frontier to change. Frost and Savarino [22] suggested portfolio selection within a Bayesian framework to deal with estimation risk and stated that using classical mean to estimate expected return and other moments of asset returns leads to suboptimal portfolio choices resulting in a loss of investor utility. Jorion [15] indicated that uncertainty about parameter values leads to suboptimal portfolio choices resulting in a loss in utility if historical average is used as a true parameter estimate. As documented in Jorion [15, 23], the James-Stein estimator is derived from the summation of components of a quadratic loss function using a shrinkage function to estimate parameter values and is used to validate the claim that sample mean is an inadmissible estimator. Effron and Morris [24] have proven that the James-Stein estimator dominates MLE with good rules of Bayesian properties. Jorion $[15,23]$ extended the work of James-Stein to a BayesStein shrinkage mean assuming that variance parameters are known.

Britten-Jones [25] used 20 years of data on 11 country stock indexes to test hypotheses about the weights of meanvariance efficient portfolios. The evidence documented that sampling error in estimates of the weights of global efficient portfolios is as large as when the return vector and variancecovariance matrix are estimated by a traditional approach. This means that the portfolio risks of the traditionally suggested efficient portfolios will be underestimated compared with that of optimal portfolio incorporating estimation risk. Taking estimation uncertainty in portfolio construction process, Michaud [4] proposed Resample Efficiency technique introducing Monte Carlo methods based on eight asset classes (Eight asset classes are Canada, France, Germany, Japan, U.K., U.S. equities, and U.S. and Euros government/corporate bonds.) of 18 years historical data. Resampled Efficiency approach solved two major drawbacks of traditional portfolio namely, concentrated and instability portfolios. Traditional portfolio optimization yields the concentrated portfolio by which some assets never enter the solution and few assets are included in the optimal portfolio composition. Instability portfolio implies that any small change in input parameters cause wide fluctuation in results. Markowitz and Usmen [17] performed experiment on portfolio performance between resampled and diffuse Bayes portfolios and reported that resampled efficient portfolios outperform those of diffuse Bayes portfolios. He [26] revised an information updating model of Treynor and Black [27] within a Bayesian framework accounting for alpha uncertainty. By varying level of overall active risk budget and centering alpha on its equilibrium level of zero, the result indicated that pension fund managers can reflects the overall confidence in the ability of active management. However, no recommendation for a better portfolio formation strategy had been made.

This study emphasizes investigating and suggesting an appropriate portfolio formation strategy by applying Bayesian shrinkage estimation to portfolio selection when uncertainty about parameter values exists. Two major portfolio formation strategies are (i) optimized portfolio within a traditional mean-variance efficient and (ii) optimized portfolio applying shrinkage asset return incorporating a factor model approach. Model and methodology are discussed in Section 2. The data and descriptive statistics of sectorial returns are discussed in Section 3. Section 4 elaborates empirical evidences. The last section is the conclusion.

\section{Model and Methodology}

Estimation risk in parameters of asset return can be treated appropriately under a Bayesian framework with either noninformative or informative prior distribution to shrink value of parameter estimate towards an equilibrium value, or grand mean. The informative prior in this study is that all asset return characteristics comply with a factor model such as, the single index model. This means that, if asset characteristics based on historical average differ from the single index model, expected predictive returns will be drawn toward the expected return suggested by the factor model. Six alternative approaches in constructing an optimal portfolio are explored. The first is traditional portfolio selection treating historical estimates as true parameters. The second alternative is an optimal portfolio based on Adjusted Beta as widely used in the industry. The common adjusted beta, as suggested by Merrill Lynch, is the weighted average between sample beta estimate and the market beta. Adjusted beta $=$ $2 / 3\left(\right.$ beta $\left._{i}\right)+(1 / 3)\left(\right.$ beta $\left._{m}\right)$. The third approach is Resampled Efficiency approach averaging optimal weights of the same ranked portfolio. The fourth portfolio formation strategy is based on Capital Asset Pricing Model (CAPM). The fifth strategy is traditional Single Index Model (SIM) allowing 
TABLE 1: Descriptive Statistics of index returns. Three periods are explored, total period ranges from January 1995 to December 2008 , the first subperiod ranges from January 1995 to December 2001, and the second subperiod ranges from January 2002 to December 2008. Numbers in parentheses are $t$-statistics.

(a)

\begin{tabular}{|c|c|c|c|c|c|c|}
\hline \multirow{2}{*}{ Country } & \multicolumn{3}{|c|}{ Alpha } & \multicolumn{3}{|c|}{ Beta } \\
\hline & Total period & $\begin{array}{c}\text { First } \\
\text { subperiod }\end{array}$ & $\begin{array}{l}\text { Second } \\
\text { subperiod }\end{array}$ & Total period & $\begin{array}{c}\text { First } \\
\text { subperiod }\end{array}$ & $\begin{array}{c}\text { Second } \\
\text { subperiod }\end{array}$ \\
\hline \multirow{2}{*}{ Turkey } & $0.03344^{* * *}$ & $0.06640^{* * *}$ & 0.00389 & $1.04746^{* * *}$ & $1.24056^{* * *}$ & $0.95713^{* * *}$ \\
\hline & $(-3.15)$ & $(-3.47)$ & $(-0.43)$ & $(-6.75)$ & $(-4.48)$ & $(-7.16)$ \\
\hline \multirow{2}{*}{$\begin{array}{l}\text { Russian } \\
\text { Federation }\end{array}$} & $0.01905^{* * *}$ & $0.03654^{* * *}$ & 0.00372 & $1.37491^{* * *}$ & $1.64265^{* * *}$ & $1.14265^{* * *}$ \\
\hline & $(-2.39)$ & $(-2.56)$ & $(-0.54)$ & $(-11.83)$ & $(-7.95)$ & $(-11.12)$ \\
\hline \multirow{2}{*}{ Hungary } & $0.00953^{*}$ & $0.02532 *$ & -0.00442 & $0.79591^{* * *}$ & $0.86536^{* * *}$ & $0.76185^{* * *}$ \\
\hline & $(-1.71)$ & $(-1.71)$ & $(-0.91)$ & $(-9.82)$ & $(-5.99)$ & $(-10.56)$ \\
\hline \multirow{2}{*}{ Mexico } & $0.00894^{* * *}$ & $0.01418^{* * *}$ & $0.00593^{*}$ & $0.69188^{* * *}$ & $0.80156^{* * *}$ & $0.58642^{* * *}$ \\
\hline & $(-2.51)$ & $(-2.23)$ & $(-1.82)$ & $(-13.30)$ & $(-8.70)$ & $(-12.07)$ \\
\hline \multirow{2}{*}{ Brazil } & $0.00892^{* * *}$ & $0.01287^{* *}$ & 0.00643 & $0.88102^{* * *}$ & $0.98651^{* * *}$ & $0.80493^{* * *}$ \\
\hline & $(-2.29)$ & $(-1.95)$ & $(-1.52)$ & $(-15.49)$ & $(-10.31)$ & $(-12.76)$ \\
\hline \multirow{2}{*}{ China } & 0.00781 & 0.00479 & 0.00869 & $0.90282^{* * *}$ & $0.79943^{* * *}$ & $0.99837^{* * *}$ \\
\hline & $(-1.08)$ & $(-0.37)$ & $(-1.20)$ & $(-8.52)$ & $(-4.30)$ & $(-9.22)$ \\
\hline \multirow{2}{*}{$\begin{array}{l}\text { South } \\
\text { Africa }\end{array}$} & $0.00648^{* * *}$ & $0.01084^{* * *}$ & 0.00366 & $0.67337^{* * *}$ & $0.74886^{* * *}$ & $0.59815^{* * *}$ \\
\hline & $(-2.09)$ & $(-2.25)$ & $(-0.92)$ & $(-14.88)$ & $(-10.73)$ & $(-10.10)$ \\
\hline \multirow{2}{*}{ India } & 0.00564 & 0.00055 & 0.00849 & $0.78962^{* * *}$ & $0.59681^{* * *}$ & $0.98873^{* * *}$ \\
\hline & $(-1.13)$ & $(-0.07)$ & $(-1.43)$ & $(-10.80)$ & $(-5.12)$ & $(-11.13)$ \\
\hline \multirow{2}{*}{ Poland } & 0.00526 & 0.01573 & -0.00379 & $0.71096^{* * *}$ & $0.72058^{* * *}$ & $0.71894^{* * *}$ \\
\hline & $(-0.91)$ & $(-1.50)$ & $(-0.76)$ & $(-8.45)$ & $(-4.73)$ & $(-9.67)$ \\
\hline \multirow{2}{*}{ Indonesia } & 0.00494 & 0.00347 & 0.00586 & $0.78745^{* * *}$ & $0.75247^{* * *}$ & $0.81547^{* * *}$ \\
\hline & $(-0.82)$ & $(-0.32)$ & $(-1.01)$ & $(-8.91)$ & $(-4.74)$ & $(-9.46)$ \\
\hline \multirow{2}{*}{ Columbia } & 0.00493 & -0.00724 & $0.01628^{* * *}$ & $0.43162^{* * *}$ & $0.33934^{* * *}$ & $0.51276^{* * *}$ \\
\hline & $(-1.04)$ & $(-0.98)$ & $(-2.74)$ & $(-6.24)$ & $(-3.18)$ & $(-5.80)$ \\
\hline \multirow{2}{*}{ Peru } & 0.00434 & 0.00131 & 0.0058 & $0.51444^{* * *}$ & $0.44296^{* * *}$ & $0.56970^{* * *}$ \\
\hline & $(-1.08)$ & $(-0.21)$ & $(-1.13)$ & $(-8.76)$ & $(-4.80)$ & $(-7.47)$ \\
\hline \multirow{2}{*}{ Pakistan } & 0.00359 & -0.00085 & 0.00701 & $0.46288^{* * *}$ & $0.53493^{* * *}$ & $0.34748^{* * *}$ \\
\hline & $(-0.46)$ & $(-0.07)$ & $(-0.73)$ & $(-4.06)$ & $(-2.93)$ & $(-2.43)$ \\
\hline \multirow{2}{*}{ Argentina } & 0.0033 & 0.00885 & 0.00422 & $0.78993^{* * *}$ & $1.01402^{* * *}$ & $0.59031^{* * *}$ \\
\hline & $(-0.54)$ & $(-0.85)$ & $(-0.70)$ & $(-8.82)$ & $(-6.70)$ & $(-6.58)$ \\
\hline \multirow{2}{*}{ Chile } & 0.0001 & -0.00123 & 0.00392 & $0.45985^{* * *}$ & $0.59743^{* * *}$ & $0.32677^{* * *}$ \\
\hline & $(-0.04)$ & $(-0.29)$ & $(-1.19)$ & $(-11.38)$ & $(-9.62)$ & $(-6.63)$ \\
\hline \multirow{2}{*}{ Malaysia } & -0.00165 & 0.00279 & -0.00245 & $0.66056^{* * *}$ & $0.90756^{* * *}$ & $0.44679^{* * *}$ \\
\hline & $(-0.34)$ & $(-0.32)$ & $(-0.78)$ & $(-9.43)$ & $(-7.14)$ & $(-8.80)$ \\
\hline \multirow{2}{*}{ Taiwan } & -0.00254 & 0.00699 & $-0.01154^{* * *}$ & $0.83599^{* * *}$ & $1.00786^{* * *}$ & $0.69489^{* * *}$ \\
\hline & $(-0.59)$ & $(-0.97)$ & $(-2.64)$ & $(-13.29)$ & $(-9.67)$ & $(-10.66)$ \\
\hline \multirow{2}{*}{ Philippines } & -0.00256 & -0.00183 & -0.00085 & $0.63556^{* * *}$ & $0.85783^{* * *}$ & $0.41319^{* * *}$ \\
\hline & $(-0.55)$ & $(-0.24)$ & $(-0.16)$ & $(-9.41)$ & $(-7.87)$ & $(-5.33)$ \\
\hline \multirow{2}{*}{ Thailand } & -0.00453 & -0.00394 & -0.00248 & $0.93898^{* * *}$ & $1.10057^{* * *}$ & $0.81091^{* * *}$ \\
\hline & $(-0.70)$ & $(-0.34)$ & $(-0.43)$ & $(-9.98)$ & $(-6.50)$ & $(-9.55)$ \\
\hline
\end{tabular}


(a) Continued.

\begin{tabular}{|c|c|c|c|c|c|c|c|c|c|}
\hline \multirow{2}{*}{ Country } & \multicolumn{3}{|c|}{ F-Stat } & \multicolumn{3}{|c|}{ Average return } & \multicolumn{3}{|c|}{ Standard deviation } \\
\hline & Total period & $\begin{array}{c}\text { First } \\
\text { subperiod }\end{array}$ & $\begin{array}{c}\text { Second } \\
\text { subperiod }\end{array}$ & Total period & $\begin{array}{c}\text { First } \\
\text { subperiod }\end{array}$ & $\begin{array}{c}\text { Second } \\
\text { subperiod }\end{array}$ & Total period & $\begin{array}{c}\text { First } \\
\text { subperiod }\end{array}$ & $\begin{array}{c}\text { Second } \\
\text { subperiod }\end{array}$ \\
\hline Turkey & $45.00^{* * *}$ & $20.07^{* * *}$ & $51.29^{* * *}$ & $3.35 \%$ & $5.79 \%$ & $0.98 \%$ & $15.59 \%$ & $19.34 \%$ & $10.37 \%$ \\
\hline $\begin{array}{l}\text { Russian } \\
\text { Federation }\end{array}$ & $135.70^{* * *}$ & $63.23^{* * *}$ & $123.66^{* * *}$ & $1.92 \%$ & $2.53 \%$ & $1.08 \%$ & $14.01 \%$ & $17.20 \%$ & $9.91 \%$ \\
\hline Hungary & $93.12^{* * *}$ & $35.99^{* * *}$ & $111.49^{* * *}$ & $0.96 \%$ & $1.94 \%$ & $0.03 \%$ & $9.06 \%$ & $10.83 \%$ & $6.75 \%$ \\
\hline Mexico & $171.37^{* * *}$ & $75.67^{* * *}$ & $145.71^{* * *}$ & $0.90 \%$ & $0.87 \%$ & $0.96 \%$ & $6.64 \%$ & $8.00 \%$ & $4.93 \%$ \\
\hline Brazil & $241.00^{* * *}$ & $106.19^{* * *}$ & $162.82^{* * *}$ & $0.90 \%$ & $0.61 \%$ & $1.14 \%$ & $7.89 \%$ & $9.08 \%$ & $6.64 \%$ \\
\hline China & $69.44^{* * *}$ & $18.30^{* * *}$ & $85.04^{* * *}$ & $0.79 \%$ & $-0.07 \%$ & $1.49 \%$ & $11.30 \%$ & $12.93 \%$ & $9.41 \%$ \\
\hline $\begin{array}{l}\text { South } \\
\text { Africa }\end{array}$ & $213.13^{* * *}$ & $115.15^{* * *}$ & $102.03^{* * *}$ & $0.65 \%$ & $0.57 \%$ & $0.74 \%$ & $6.14 \%$ & $6.77 \%$ & $5.40 \%$ \\
\hline India & $113.41^{* * *}$ & $26.21^{* * *}$ & $123.91^{* * *}$ & $0.57 \%$ & $-0.35 \%$ & $1.46 \%$ & $8.48 \%$ & $8.38 \%$ & $8.57 \%$ \\
\hline Poland & $68.66^{* * *}$ & $22.42^{* * *}$ & $93.56^{* * *}$ & $0.53 \%$ & $1.08 \%$ & $0.07 \%$ & $8.95 \%$ & $10.75 \%$ & $6.62 \%$ \\
\hline Indonesia & $75.96^{* * *}$ & $22.49^{* * *}$ & $89.44^{* * *}$ & $0.50 \%$ & $-0.17 \%$ & $1.09 \%$ & $9.56 \%$ & $11.21 \%$ & $7.59 \%$ \\
\hline Columbia & $39.20^{* * *}$ & $10.12^{* * *}$ & $33.63^{* * *}$ & $0.50 \%$ & $-0.96 \%$ & $1.95 \%$ & $6.84 \%$ & $7.07 \%$ & $6.39 \%$ \\
\hline Peru & $72.47^{* * *}$ & $23.05^{* * *}$ & $55.73^{* * *}$ & $0.44 \%$ & $-0.17 \%$ & $0.93 \%$ & $6.31 \%$ & $6.54 \%$ & $6.02 \%$ \\
\hline Pakistan & $15.16^{* * *}$ & $8.59^{* * *}$ & $5.91^{* * *}$ & $0.36 \%$ & $-0.45 \%$ & $0.92 \%$ & $10.63 \%$ & $12.01 \%$ & $9.01 \%$ \\
\hline Argentina & $81.50^{* * *}$ & 44.89 & 43.36 & $0.34 \%$ & $0.19 \%$ & $0.79 \%$ & $9.65 \%$ & $11.78 \%$ & $6.75 \%$ \\
\hline Chile & 132.26 & $92.45^{* * *}$ & $43.97^{* * *}$ & $0.01 \%$ & $-0.53 \%$ & $0.60 \%$ & $4.79 \%$ & $5.67 \%$ & $3.72 \%$ \\
\hline Malaysia & $92.46^{* * *}$ & $51.01^{* * *}$ & $77.43^{* * *}$ & $-0.16 \%$ & $-0.34 \%$ & $0.03 \%$ & $7.72 \%$ & $10.13 \%$ & $4.31 \%$ \\
\hline Taiwan & $177.67^{* * *}$ & $93.60^{* * *}$ & $113.59^{* * *}$ & $-0.25 \%$ & $0.01 \%$ & $-0.72 \%$ & $8.02 \%$ & $9.54 \%$ & $6.13 \%$ \\
\hline Philippines & $87.64^{* * *}$ & $62.00^{* * *}$ & $28.44^{* * *}$ & $-0.25 \%$ & $-0.77 \%$ & $0.17 \%$ & $7.44 \%$ & $9.03 \%$ & $5.47 \%$ \\
\hline Thailand & $101.92^{* * *}$ & $42.22^{* * *}$ & $91.16^{* * *}$ & $-0.44 \%$ & $-1.15 \%$ & $0.26 \%$ & $10.57 \%$ & $13.05 \%$ & $7.51 \%$ \\
\hline
\end{tabular}

$*, * *$, and $* * *$ indicate significant at $10 \%, 5 \%$, and $1 \%$, respectively.

(b)

\begin{tabular}{|c|c|c|c|}
\hline \multirow{2}{*}{ Country } & \multicolumn{3}{|c|}{ Information ratio } \\
\hline & Total period & First subperiod & Second subperiod \\
\hline Turkey & 295.7651 & 181.6571 & 48.3165 \\
\hline Russian Federation & 300.5328 & 179.6849 & 78.2327 \\
\hline
\end{tabular}


(b) Continued.

\begin{tabular}{lccc}
\hline Country & Total period & $\begin{array}{c}\text { Information ratio } \\
\text { First subperiod }\end{array}$ & Second subperiod \\
\hline Hungary & 308.6629 & 255.3434 & -188.4281 \\
Mexico & 703.1102 & 350.4998 & 557.6252 \\
Brazil & 587.5088 & 294.6973 & 358.7845 \\
China & 148.1109 & 28.7707 & 164.5916 \\
South Africa & 674.1654 & 467.1711 & 231.4469 \\
India & 224.6173 & 8.5055 & 239.0569 \\
Poland & 158.2308 & 142.5708 & -152.1374 \\
Indonesia & 134.6862 & 28.8828 & 174.9011 \\
Columbia & 219.6666 & -133.5755 & 462.2749 \\
Peru & 268.1393 & 32.2578 & 221.1845 \\
Pakistan & 58.9806 & -5.3772 & 76.1875 \\
Argentina & 87.5264 & 81.0338 & 116.5557 \\
Chile & 13.5179 & -66.9743 & 358.3304 \\
Malaysia & -71.5389 & 36.3157 & -210.6151 \\
Taiwan & -136.7961 & 135.1681 & -602.6266 \\
Philippines & -119.5397 & -32.4259 & -31.299 \\
Thailand & -109.0524 & -28.7929 & -76.1938 \\
\hline
\end{tabular}

TABLE 2: Portfolio performance of alternative estimation methods: Sharpe’s Ratio.

\begin{tabular}{|c|c|c|c|c|c|c|}
\hline \multirow{3}{*}{ Strategy } & \multicolumn{6}{|c|}{ Monthly excess portfolio return } \\
\hline & \multicolumn{3}{|c|}{ Exante } & \multicolumn{3}{|c|}{ Expost } \\
\hline & Total period (TP) & Subperiod 1 (SP1) & Subperiod $2(\mathrm{SP} 2)$ & Total period (TP) & Subperiod 1 (SP1) & Subperiod 2 (SP2) \\
\hline Mean-Variance & $1.84 \%$ & $2.74 \%$ & $1.26 \%$ & $-5.43 \%$ & $-6.38 \%$ & $-1.46 \%$ \\
\hline $\mathrm{AB}$ & $-0.02 \%$ & $-0.47 \%$ & $-0.44 \%$ & $-2.31 \%$ & $1.50 \%$ & $-2.47 \%$ \\
\hline REF & $2.04 \%$ & $2.32 \%$ & $1.21 \%$ & $-2.84 \%$ & $3.01 \%$ & $-1.44 \%$ \\
\hline CAPM & $-0.02 \%$ & $-0.36 \%$ & $0.54 \%$ & $-2.31 \%$ & $1.50 \%$ & $-2.80 \%$ \\
\hline SIM & $1.94 \%$ & $2.42 \%$ & $1.27 \%$ & $-3.44 \%$ & $-4.03 \%$ & $1.65 \%$ \\
\hline \multirow[t]{2}{*}{ BSIM } & $2.39 \%$ & $2.67 \%$ & $2.70 \%$ & $-1.07 \%$ & $3.08 \%$ & $-3.21 \%$ \\
\hline & \multicolumn{6}{|c|}{ Monthly excess portfolio risk } \\
\hline \multirow[t]{2}{*}{ Strategy } & & Exante & & & Expost & \\
\hline & Total period (TP) & Subperiod 1 (SP1) & Subperiod $2(\mathrm{SP} 2)$ & Total period (TP) & Subperiod 1 (SP1) & Subperiod 2 (SP2) \\
\hline Mean-Variance & $8.84 \%$ & $10.56 \%$ & $4.36 \%$ & $0.93 \%$ & $1.37 \%$ & $0.20 \%$ \\
\hline $\mathrm{AB}$ & $2.75 \%$ & $3.39 \%$ & $2.01 \%$ & $2.75 \%$ & $7.73 \%$ & $3.37 \%$ \\
\hline REF & $0.29 \%$ & $0.25 \%$ & $0.19 \%$ & $1.05 \%$ & $0.87 \%$ & $1.38 \%$ \\
\hline CAPM & $2.75 \%$ & $3.39 \%$ & $3.60 \%$ & $2.75 \%$ & $7.73 \%$ & $3.37 \%$ \\
\hline SIM & $9.22 \%$ & $10.85 \%$ & $5.96 \%$ & $9.21 \%$ & $10.77 \%$ & $5.26 \%$ \\
\hline \multirow[t]{2}{*}{ BSIM } & $7.59 \%$ & $9.22 \%$ & $5.27 \%$ & $7.56 \%$ & $9.18 \%$ & $5.26 \%$ \\
\hline & \multicolumn{6}{|c|}{ Sharpe's Ratio } \\
\hline \multirow[t]{2}{*}{ Strategy } & & Exante & & & Expost & \\
\hline & Total period (TP) & Subperiod 1 (SP1) & Subperiod 2 (SP2) & Total period (TP) & Subperiod 1 (SP1) & Subperiod 2 (SP2) \\
\hline Mean-Variance & 0.1949 & 0.2189 & 0.2824 & -10.1536 & -1.5029 & -10.4492 \\
\hline $\mathrm{AB}$ & -0.0093 & 0.2410 & 0.2038 & -1.1015 & -1.6834 & -0.3573 \\
\hline REF & 0. 2089 & 0.2622 & 0.3830 & -0.3681 & 0.1648 & -0.9629 \\
\hline CAPM & -0.0075 & -0.1337 & 0.1974 & -1.1015 & 0.3107 & -1.2359 \\
\hline SIM & 0.2038 & 0.2067 & 0.2113 & -0.3573 & -0.3198 & $0.3127^{*}$ \\
\hline BSIM & $0.3147^{*}$ & $0.2900^{*}$ & $0.5111^{*}$ & $-0.1409^{*}$ & $0.3357^{*}$ & -0.6091 \\
\hline
\end{tabular}

Note: Ex ante and ex-post-portfolio monthly return and risk are reported. Total sample periods range from January 1995 to December 2008 . Two out-ofsample periods are January 1996 and January 2009.

* denotes the highest Sharpe's Ratio compared among different portfolio strategies. 
for asset mispricing. The sixth alternative is an empirical Bayesian approach with an informative prior incorporating a factor model namely, Bayesian Single Index Model (BSIM).

Given that an investor has $T$ observations for each individual $N$ traded assets. Let $R$ be asset return matrix with dimension $T \times N ; m_{i}$ is a vector of expected returns of securities, and $\Sigma$ is a population variance-covariance matrix of security returns. Returns are calculated with both continuous (difference of log price) and discrete returns (ratio of adjusted price difference). Results based on continuous and discrete return calculations are not different. Alternative models discussed below suggest that, by specifying different beliefs in prior distribution, portfolio selection yields different results.

2.1. Traditional Mean-Variance Approach, Resampled Efficiency Frontier (REF), Capital Asset Pricing Model (CAPM), and Traditional Single Index Model (SIM) Approach. Within a traditional mean-variance portfolio selection framework, sample estimates are treated as true parameters. This approach can be called the certainty equivalence method, and estimation risk is not taken into account. Hence, the sample mean vector and sample covariance matrix are major inputs in the portfolio optimization process as shown in (1).

$$
\begin{gathered}
m_{i}=\frac{\iota^{\prime} \widetilde{R}_{i}}{T}, \quad i=1,2, \ldots, n, \\
S=\frac{\left(\widetilde{R}_{i}^{\prime}-m_{i}\right)^{\prime}\left(\widetilde{R}_{j}^{\prime}-m_{j}\right)}{T-1}, \quad i, j=1,2, \ldots, n,
\end{gathered}
$$

where $m_{i}=$ sample mean vector, $S=$ sample variancecovariance matrix, $\widetilde{R}_{i}^{\prime}=\left(\tilde{r}_{i 1}, \tilde{r}_{i 2}, \ldots, \tilde{r}_{i T}\right)=$ excess return of each individual asset, $T=$ number of observations, $n=$ number of assets, $\iota^{\prime}=(1,1, \ldots, 1)$.

Resampled Efficiency Frontier (REF) generates observed asset returns by Monte Carlo approach from two major parameters given that asset returns follow multivariate normal distribution. After each set of observed returns are generated, efficient frontier spanning from minimum variance portfolio to maximum return portfolio is constructed using traditional mean variance optimization approach. One hundred and one portfolios are determined on each efficient frontier, and corresponding allocated weights are recorded. The distance between minimum and maximum returns is equally split. Repeating the same processes for 500 times, we have 500 different sets of parameter inputs and 500 efficient frontiers. Number of observed returns is 500 sets as suggested in Markowitz and Usmen [17]. The original set of inputs is denoted as $\left(\mu_{0}, \Omega_{0}\right)$ and obtained input sets are denoted as $\left(\hat{\mu}_{1}, \widehat{\Omega}_{1}\right),\left(\hat{\mu}_{2}, \widehat{\Omega}_{2}\right), \ldots,\left(\hat{\mu}_{500}, \widehat{\Omega}_{500}\right)$. Portfolios Ranked 1 are the minimum variance portfolio and portfolios ranked 101 are maximum return portfolios. Averaging optimal weights of each ranked portfolio from 500 efficient frontiers, we obtain average resample weights. Portfolio risk and return are calculated in the last step.
Within the Capital Asset Pricing Model (CAPM) and a single index model approach, asset return generating process is stated as shown in (2) and (3), respectively,

$$
\begin{gathered}
R=R_{m} \beta, \\
R=R_{m} C+U,
\end{gathered}
$$

where $R$ = vector of expected excess return on each individual asset, $R_{m t}=$ vector of expected excess market index return, $\beta$ $=$ vector of beta coefficient, $C \equiv\left[\begin{array}{l}\alpha^{\prime} \\ \beta^{\prime}\end{array}\right]=$ coefficient vector, $U \sim N\left(0, \Sigma \otimes I_{T}\right)=$ residual matrix containing residual term of each asset, $\varepsilon_{i}, I_{T}=$ identity matrix with rank $T$.

Residual terms in matrix $U$ are assumed to be independent, serially uncorrelated, and homoskedastic. Two crucial parameters, expected return and variance-covariance matrix for an individual asset, in efficient portfolio formation are shown below:

$$
\begin{gathered}
E\left(R_{i}\right)=R_{m} C, \\
\sigma_{i}^{2}=\beta_{i}^{\prime} \sigma_{m}^{2} \beta_{i}+\sigma_{\varepsilon_{i}}^{2}, \\
\sigma_{i j}=\beta_{i}^{\prime} \sigma_{m}^{2} \beta_{j}^{\prime}+\sigma_{\varepsilon_{i}}^{2},
\end{gathered}
$$

where: $\sigma_{i}^{2}=$ variance of an individual asset, $\sigma_{m}^{2}=$ variance of market index portfolio, $\sigma_{\mathcal{E}_{i}}^{2}=$ variance of residual terms, $\sigma_{i j}=$ covariance of two individual assets.

If market efficient hypothesis holds, alpha or the intercept term in the single index model will be zero. When alpha has a nonzero value, it indicates mispricing for the set of traded assets. Portfolio managers can outperform the market by determining and investing in nonzero alpha assets.

2.2. Bayesian Single Index Model (BSIM). Within this framework, the objective is to determine posterior distribution of parameter estimates; likelihood function and prior distribution must be determined via the conjugate function. He [26] suggests that the selected conjugate function with return generating process is given by (5), and prior distribution of the coefficient vector, $C$, and the variance-covariance matrix, $\Sigma$, are given in (6)

$$
\begin{aligned}
& p(R \mid C, \Sigma) \\
& \propto|\Sigma|^{-T / 2} \exp \left\{-\frac{1}{2} \operatorname{tr}(R-X C)^{\prime}(R-X C) \Sigma^{-1}\right\} \\
& \propto|\Sigma|^{-T / 2} \exp \left\{-\frac{1}{2} \operatorname{tr}\left[S+(C-\hat{C})^{\prime} V_{0}^{-1}(C-\hat{C}) \Sigma^{-1}\right]\right\}
\end{aligned}
$$

where $S \equiv(R-X C)^{\prime}(R-X C), \widehat{C} \equiv\left(X^{\prime} X\right)^{-1} X^{\prime} R$,

$$
p(C, \Sigma)=p(C \mid \Sigma) p(\Sigma)
$$

where $p(C \mid \Sigma) \sim N\left(C_{0}, \Sigma \otimes V_{0}\right) \propto|\Sigma|^{-(K+1) / 2} \exp \{-(1 /$ 2) $\left.\operatorname{tr}\left(C-C_{0}\right)^{\prime} V_{0}^{-1}\left(C-C_{0}\right) \Sigma^{-1}\right\}, C \equiv\left[\begin{array}{l}\alpha^{\prime} \\ \beta^{\prime}\end{array}\right]=$ coefficient vector, $V_{0}^{-1} \equiv\left[\begin{array}{cc}S^{2} / \sigma_{\alpha}^{2} & 0 \\ 0 & 0\end{array}\right], p(\Sigma) \sim \operatorname{IW}\left(H_{0}, \nu_{0}\right) \propto$ $|\Sigma|^{-\left(\nu_{0}+N+1\right) / 2} \exp \left\{-(1 / 2) \operatorname{tr} H_{0} \Sigma^{-1}\right\}$, IW $=$ Inverted-Wishart 
TABle 3: Portfolio Performance of Alternative Estimation Methods: Expected Utility.

\begin{tabular}{lccccccccc}
\hline & \multicolumn{3}{c}{ Total period (TP) } & \multicolumn{3}{c}{ Expected Utility } \\
Strategy & \multicolumn{3}{c}{ Subperiod 1 (SP1) } & \multicolumn{4}{c}{ Subperiod 2 (SP2) } \\
& $A=0.5$ & $A=1$ & $A=2$ & $A=0.5$ & $A=1$ & $A=2$ & $A=0.5$ & $A=1$ & $A=2$ \\
\hline Mean-Variance & 0.00553 & 0.00196 & -0.00265 & 0.00452 & 0.00021 & -0.00641 & 0.01683 & 0.01376 & 0.00865 \\
AB & -0.00052 & -0.00076 & -0.00125 & -0.00546 & -0.00607 & -0.00691 & 0.00627 & 0.00577 & 0.00511 \\
REF & 0.00445 & 0.00088 & -0.00368 & 0.00239 & -0.00206 & -0.00820 & 0.01317 & 0.01083 & 0.00669 \\
CAPM & -0.00048 & -0.00072 & -0.00118 & -0.00419 & -0.00494 & -0.00593 & 0.00658 & 0.00594 & 0.00512 \\
SIM & 0.00603 & -0.00149 & -0.01288 & 0.00721 & -0.00157 & -0.01845 & 0.0169 & 0.0135 & 0.00669 \\
BSIM & $0.06309^{*}$ & $0.05967^{*}$ & $0.05283^{*}$ & $0.06488^{*}$ & $0.06001^{*}$ & $0.05026^{*}$ & $0.06736^{*}$ & $0.06547^{*}$ & $0.06170^{*}$ \\
\hline
\end{tabular}

* denotes the highest Expected Utility given three different risk aversion level $(A), A=0.5,1$, and 2, compared among different portfolio strategies.

Distribution, $H_{0}=S^{2} I_{N}, S^{2}=$ Average of diagonal elements of the sample residual error matrix.

Posterior distribution can be determined by collecting terms from the product of likelihood function and prior distribution as shown below:

$$
\begin{aligned}
p(C, \Sigma \mid R) \propto & p(R \mid C, \Sigma) p(C \mid \Sigma) p(\Sigma) \\
\propto & |\Sigma|^{-\left(T+K+1+\nu_{0}+N+1\right) / 2} \\
& \times \exp \left\{-\frac{1}{2} \operatorname{tr}\left[(C-\tilde{C})^{\prime} \tilde{V}^{-1}(C-\tilde{C})+\tilde{H}\right]\right\}
\end{aligned}
$$

where $\tilde{C} \equiv\left(V_{0}^{-1}+X^{\prime} X\right)^{-1}\left(V_{0}^{-1} C_{0}+X^{\prime} R\right), \quad \tilde{V} \equiv$ $\left(V_{0}^{-1}+X^{\prime} X\right)^{-1}, \tilde{H} \equiv H_{0}+S+C_{0}^{\prime} V_{0}^{-1} C_{0}+\hat{C}^{\prime} X^{\prime} X \widehat{C}-\widetilde{C}^{\prime} V^{-1} \widetilde{C}$.

From the posterior distribution above, two indications can be made. Given historical series of asset returns, firstly, joint distribution of two major parameters, $p(C, \Sigma \mid R)$, follows multivariate normal distribution with posterior mean $\widetilde{C}$ and posterior variance $\Sigma \otimes \widetilde{V}$. Secondly, distribution of variance-covariance matrix, $p(\Sigma \mid R)$, has an invertedWishart distribution with degree of freedom $\tilde{v}=T+\nu_{0}$ and a scale matrix $\tilde{H}$.

Construct an optimal portfolio based on the single index model; if an investor has a strong belief that the market is efficient and there is no mispricing, alpha will be zero and the model converges to the equilibrium model CAPM. In real world, there are some room to make abnormal return by searching assets with nonzero alpha to capitalize on mispricing phenomenon. Shrinkage Bayesian model presented in this paper suggests that if mispricing exists, estimation risk in parameter estimates, $\alpha$ and $\beta$, should be taken into account by shrinking the two estimates to its equilibrium value with the Bayesian adjustment factor shown below:

$$
\widetilde{C} \equiv\left[\begin{array}{l}
\alpha^{\prime} \\
\beta^{\prime}
\end{array}\right]=\left(V_{0}^{-1}+X^{\prime} X\right)^{-1} X^{\prime} R .
$$

To form a portfolio that incorporates estimation risk, we apply Bayesian concepts to alleviate the effect of estimation risk. As discussed in Section 1, both informative and noninformative prior distributions are applied in constructing the optimal portfolio. Incorporating estimation risk in a portfolio formation strategy would improve portfolio performance. To validate this claim, an empirical test was performed.

2.3. Portfolio Performance. Two portfolio performance measurements are used in this study, namely, expected utility and Sharpe's Ratio. The ultimate goal of an investor is maximizing one's terminal wealth leading to maximize one's expected utility. The first portfolio performance measurement is stated below:

$$
\operatorname{Max} \mathrm{EU}=E-\lambda(V),
$$

where $\mathrm{EU}=$ expected utility of each portfolio, $E=$ portfolio return, $\lambda=$ coefficient of degree of risk aversion: $0.5,1,2, V$ $=$ portfolio variance.

Given a different level of degree of risk aversion, expected utility of each portfolio from each strategy will be assessed. Among all portfolio formation strategies, the portfolio possessing the highest expected utility is considered as the best performance portfolio.

Since only two moments are used in constructing an optimal portfolio, the second portfolio performance measurement is Sharpe's ratio. The best strategy among six strategies will yield highest Sharpe's ratio. Ex ante portfolio performance measurements are compared to out-of-sample ex-post-portfolio performance measurements. The better portfolio formation strategy leads to a higher expected utility and Sharpe's ratio for both in- and out-of-sample data.

\section{Data and Descriptive Statistics}

Data used in this study are monthly index returns of 19 emerging markets adjusted for dividend and emerging market price index. list of countries in emerging markets based on FTSE emerging market list. Data are obtained from Data Stream. Emerging market price index is obtained from Data Stream under DS Mnemonic TOTMKEK in U.S. dollar unit. Nineteen emerging countries with complete data are Argentina, Brazil, Chile, China, Columbia, Hungary, India, Indonesia, Malaysia, Mexico, Pakistan, Peru, the Philippines, Poland, Russian Federation, South Africa, Taiwan, Thailand, and Turkey. Quotations of each market index are based on the same currency, U.S. dollar. Periods covered in this study incorporate long range of time from 1995 to 
2008 which incorporates global crises. Divided into two subperiods, 1995-2001 and 2002-2008, data had covered global financial crises. Global financial crises covered the followings events, Asian financial crisis in 1997, Long-Term Capital Management (LTCM) crisis in Japan and Russia in 1998, IT bubble burst and dot com crisis in 2000, bankruptcy scandal of ENRON in late 2001, bond market crisis in 2003, and US subprime crisis in 2008, etc. Sample period is total sample period, and two subperiods. Total sample period ranges from January 1995 to December 2008. The first subperiod ranges from January 1995 to December 2001 and the second subperiod ranges from January 2002 to December 2008.

Descriptive statistics are in Tables 1(a) and 1(b). Emerging market indexes are drawn from Data Stream, DSTOTMK, which are available during 1990s with the same base of 100 . Table 1 (b) reports information ratio, $\alpha_{i} / \sigma_{\alpha_{i}}^{2}$, of each country for all periods studied. Descriptive results from Table 1(a) indicate that all emerging markets exhibit nonzero alpha with positive beta coefficient. Only a few countries with nonzero alpha are statistically significantly different from zero while all beta coefficients are statistically significantly different from zero. This could be interpreted that emerging markets risk and expected return relationship conform to modern portfolio theory and there is mispricing in some emerging countries. Fund managers can insert their own belief in determining mispricing countries and recognize the abnormal return from such portfolio formation strategy. Moreover, the average returns in each subperiod are not significantly different. Based on total sample period, average monthly index returns range from -0.44 percent to 3.35 percent, from -1.15 percent to 5.79 percent for the first subperiod, and from -0.72 percent to 1.95 percent for the second subperiods. Unlike average index return, standard deviations or risk levels for each country are significantly different and larger than average return. Based on total sample index return, standard deviations range from 4.79 percent to 15.59 percent, from 5.67 percent to 19.33 percent in the first subperiod, and from 3.72 percent to 10.37 percent in the second subperiod.

The information ratio, as shown in Table 1(b), indicates that the mispricing of each country is large as each value differs from zero considerably. For the total sample period, information ratio ranges from -136.796 (Taiwan) to 703.11 (Mexico), from -133.575 (Colombia) to 467.17 (South Africa) for the first subperiod, and from -602.626 (Taiwan) to 462.27 (Colombia). It can be observed that the spread of variation of information ratio was wider in the second subperiod reflecting the recent subprime financial crisis. The high value of the information ratio is the characteristic which indicates that the shrinkage Bayesian would apply.

\section{Empirical Evidence}

Theoretically, an ideal estimator would yield the same result as the true or future observed value. The only situation in which an ideal estimator would exist is when there is perfect information for an estimated parameter. Practically, an ideal estimator is impossible due to lack of perfect information. Thus, a good estimator is the best that can be expected. The six different estimating strategies are traditional meanvariance (EV), Adjusted Beta (AB), Resampled Efficient Frontier (REF), Capital Asset Pricing Model (CAPM), Single Index Model (SIM), and Single Index Model incorporating shrinkage Bayesian factor namely, Bayesian Single Index Model (BSIM).

In this study, the realized or observed industrial index return is treated as the true average return. Estimation error or mean squares forecast error is defined as the difference between true average return and estimated mean return for each sector. Estimated values from each strategy are defined as exante estimates and observed or realized returns are defined as expost values.

For each period, optimal weights are computed for each strategy. Ex-ante-portfolio return is computed for the following out of sample month. The first subperiod, ranging from January 1995 to December 2001, is the base window for the optimal weights of the first period. Exante portfolio returns are computed and recorded for the next period, which is January 2002. Observed out of sample or expost return in January 2002 for each country is recorded based on the optimal weights from the exante portfolio. The same process is repeated for the second subperiod which ranges from January 2002 to December 2008. The expost return is out of the sample observed in January 2009. From these exante and realized monthly returns and average portfolio risk, the expected utility and Sharpe's ratios of those portfolios are compared. A better portfolio strategy would yield a higher expected utility and Sharpe's ratio and lower differences between ex-ante- and ex-post-average values.

Table 2 shows that every portfolio strategy always overestimates true parameter values. Ex-ante-average excess portfolio returns are higher than those of expost averages for all strategies. Except for the first subperiod, portfolios formed by adjusted beta approach (AB), resampled efficiency frontier (REF), market equilibrium hypothesis approach (CAPM), and Bayesian approach (BSIM) outperform the forecast. Ex ante excess portfolio returns are expected to be -0.47 percent, 2.32 percent, -0.36 percent, and 2.67 percent per month for portfolios formed by AB, REF, CAPM, and BSIM, respectively whereas the ex-post-excess portfolio returns are 1.50 percent, 3.01 percent, 1.50 percent, and 3.08 percent per month. The Bayesian Shrinkage Portfolio incorporating single index model strategy (BSIM) is expected to have the largest average excess portfolio return compared with other strategies. Ex-ante-average excess portfolio returns for BSIM are 2.39 percent per month or 28.68 percent per annum, 2.67 percent per month or 32.04 percent per annum, and 2.70 percent per month or 32.40 percent per annum in total period, the first subperiod, and the second subperiod, respectively. The lowest ex-ante-average excess portfolio returns are portfolio constructed based on CAPM in all studies periods. Ex-post-average excess portfolio return for the BSIM portfolio outperforms those of other strategies in total sample period and the first subperiod. For total sample period, BSIM yields minimum loss of 1.07 percent per month or loss of 12.84 percent per annum whereas expost 
returns of other strategies yield larger loss ranges from 2.31 percent per month or 27.72 percent per annum (CAPM strategy) to 5.43 percent per month or 65.16 percent per annum (EV strategy).

Among six portfolio formation strategies, traditional portfolio approach (EV) has the largest variation as its monthly excess portfolio risk fluctuates widely while other formation strategies have intact ex-ante- and ex-postportfolio risks. As documented, the exante monthly portfolio risk of EV portfolios ranges from 8.84 percent, 10.56 percent, and 4.36 percent to 0.93 percent, 1.37 percent, and 0.20 percent in total sample period, the first subperiod, and the second subperiod, respectively. The lowest deviation between ex ante and ex-post-average excess portfolio risk is that of BSIM.

The Bayesian Single Index Model (BSIM) or Bayesian Portfolio incorporating a factor model performs best on an exante and expost basis. From exante Sharpe's Ratio, BSIM produces the largest Sharpe's Ratio and the portfolio constructed based on CAPM approach has the lowest ratio. BSIM's exante Sharpe's Ratios are the largest at 0.31, 0.29, and 0.511 in the total sample period, first subperiod, and second subperiod, respectively. The traditional meanvariance efficient portfolio approach $(\mathrm{EV})$ yields the lowest Sharpe's Ratio of $-10.15,-1.50$, and -10.45 based on expost average in each of sample period. Among optimized portfolio strategies, it can be concluded that the expost performance of the Bayesian portfolio approach exceeded that of the traditional approach.

An alternative measure of portfolio performance is the expected utility taking into account portfolio risk, return, and degrees of risk aversion. Three degrees of risk aversion are used to explore effect of investor's risk preference on portfolio performance. The higher the expected utility indicates the better performance of a portfolio. Table 3 exhibits maximum expected utility from different portfolio formation strategy given different degree of risk aversion (A), $A=0.5,1$, and 2. Portfolios constructed by BSIM approach yield the highest expected utility for all levels of risk aversion degrees. Results from Table 3 reassure that allowing for mispricing in asset prices in Bayesian portfolio formation strategy; BSIM portfolios outperform those of traditional approaches.

\section{Conclusion}

Empirical results indicate that when estimation uncertainty is taken into account, the shrinkage Bayesian strategy incorporating single index model (BSIM) outperforms the Traditional portfolio selection strategy such as mean-variance efficient, Adjusted Beta model, Resampled Efficient Frontier model, CAPM, and single index model based on both exante and expost performances. This study not only demonstrates the benefits from using shrinkage estimators to alleviate estimation uncertainty problem but also suggests an appropriate portfolio selection strategy, namely, an optimized portfolio incorporating a single index model or BSIM. Shrinkage Bayesian model presented in this paper suggests that if mispricing exists, estimation risk in parameter estimates, $\alpha$ and $\beta$, should be taken into account by shrinking the two estimates to its equilibrium value with the Bayesian adjustment factor. The major contribution of this study is that allowing for asset mispricing and applying Bayesian shrinkage adjusted factor to each asset's alpha given that alpha will be shrunk toward market equilibrium condition or at zero alpha value, a single factor namely, excess market return is adequate in alleviating estimation uncertainty.

\section{Acknowledgments}

This paper is supported by Thailand Research Funds (TRF). The authors thank anonymous referees for constructive and useful comments. The authors thank the reviewers for comments regarding hyperinflation in some emerging markets during 1990s and price jumps during crisis period. Since samples in this study are total market adjusted price indexes, considered as big portfolios containing large amount of assets, and index returns are calculated in percentage during long range of time, therefore, hyperinflation in some emerging markets has insignificant effect on market return indexes. Moreover, the objective of this study is to point out that by incorporating new information, price jumps during financial market crises, in asset pricing via Bayesian adjusted factor we could improve portfolio performance.

\section{References}

[1] H. Markowitz, "Portfolio selection," Journal of Finance, vol. 7, pp. 77-91, 1952.

[2] W. Sharpe, "Capital asset prices: a theory of market equilibrium under condition of risk," Journal of Finance, vol. 19, pp. 425-442, 1964.

[3] E. J. Elton, M. Gruber, and M. Padberg, "Simple criteria for optimal portfolio selection," Journal of Finance, vol. 31, pp. 1341-1357, 1976.

[4] R. Michaud, Efficient Asset Management, Harvard Business School Press, New York, NY, USA, 1998.

[5] A. Kraus and R. Litzenberger, "Skewness preference and the valuation of risk assets," Journal of Finance, vol. 31, pp. 10851110, 1976.

[6] Y. Kroll, H. Levy, and H. Markowitz, "Mean-variance versus direct utility maximization," Journal of Finance, vol. 39, pp. 921-931, 1984.

[7] P. Chunhachinda, K. Dandapani, S. Hamid, and A. J. Prakash, "Portfolio selection and skewness: evidence from international stock markets," Journal of Banking and Finance, vol. 21, no. 2, pp. 143-167, 1997.

[8] P. Chunhachinda, "Performance measure of global stock markets when return distributions are asymmetric," International Journal of Business Research, vol. 12, pp. 19-37, 1997.

[9] C. M. Stein, "Confidence sets for the mean of a multivariate normal distribution," Journal of the Royal Statistical Society. Series B, vol. 24, pp. 265-296, 1962.

[10] A. Karolyi, "A Bayesian approach to modeling stock return volatility for option valuation," Journal of Financial and Quantitative Analysis, vol. 28, pp. 579-594, 1993.

[11] C. Barry, "Portfolio analysis under uncertain means, variances, and covariances," Journal of Finance, vol. 29, pp. 515-522, 1974. 
[12] R. W. Klein and V. S. Bawa, "The effect of estimation risk on optimal portfolio choice," Journal of Financial Economics, vol. 3, no. 3, pp. 215-231, 1976.

[13] S. Brown, "The effect of estimation risk on capital market equilibrium," Journal of Financial and Quantitative Analysis, vol. 14, pp. 215-220, 1979.

[14] S. Chen and S. Brown, "Estimation risk and simple rules for optimal portfolio selection," Journal of Finance, vol. 38, pp. 1087-1093, 1983.

[15] P. Jorion, "Bayes-Stein estimation for portfolio analysis," Journal of Financial and Quantitative Analysis, vol. 21, pp. 279292, 1986.

[16] J. Horst, F. Roon, and B. Werker, "Incorporation Estimation Risk in Portfolio Choice," Tilburg University, Center for Economic Research Discussion Paper No. 65, 2002, http://papers.ssrn.com/sol3/paper.cfm?abstract_id=244695.

[17] H. Markowitz and N. Usmen, "Resampled frontiers versus diffuse bayes: an experiment," Journal of Investment Management, vol. 1, pp. 9-25, 2003.

[18] R. Michaud, "A practical framework for portfolio choice," in The World of Risk Management, H. G. Fong, Ed., World Scientific, Hackensack, NJ, USA, 2006.

[19] N. G. Polson and B. V. Tew, "Bayesian portfolio selection: an empirical analysis of the S\&P 500 index 1970-1996," Journal of Business and Economic Statistics, vol. 18, no. 2, pp. 164-173, 2000.

[20] L. Pástor, "Portfolio selection and asset pricing models," Journal of Finance, vol. 55, no. 1, pp. 179-223, 2000.

[21] V. S. Bawa, J. Stephen, and R. Klein, Studies in Bayesian Econometrics: Estimation Risk and Optimal Portfolio Choice, North-Holland, New York, NY, USA, 1979.

[22] P. Frost and J. Savarino, "An empirical Bayes approach to efficient portfolio selection," Journal of Financial and Quantitative Analysis, vol. 21, pp. 293-305, 1986.

[23] P. Jorion, "Bayesian and CAPM estimators of the means: implications for portfolio selection," Journal of Banking and Finance, vol. 15, no. 3, pp. 717-727, 1991.

[24] B. Efron and C. Morris, "Stein's estimation rule and its competitors," Journal of American Statistics Association, vol. 68, pp. 117-130, 1973.

[25] M. Britten-Jones, "The sampling error in estimates of meanvariance efficient portfolio weights," Journal of Finance, vol. 54, no. 2, pp. 655-671, 1999.

[26] Z. He, "Incorporating alpha uncertainty into portfolio decisions: a Bayesian revisit of the Treynor-Black model," Journal of Asset Management, vol. 8, pp. 161-175, 2007.

[27] J. L. Treynor and F. Black, "How to use security analysis to improve portfolio selection," Journal of Business, vol. 46, pp. 66-88, 1973. 


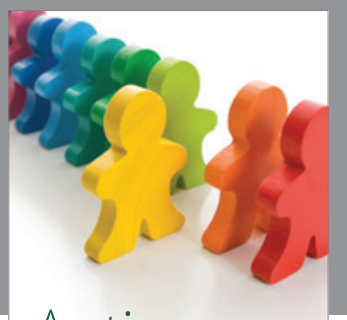

Autism

Research and Treatment
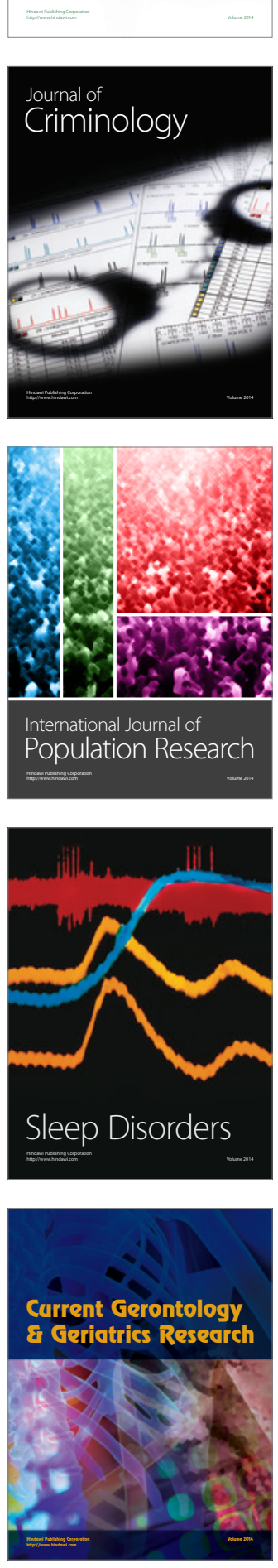
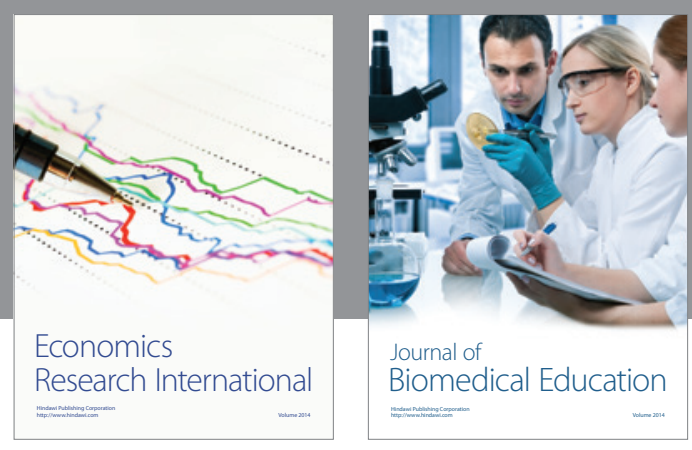

Journal of

Biomedical Education

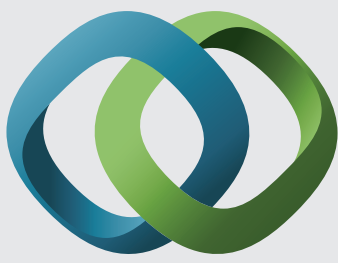

\section{Hindawi}

Submit your manuscripts at

http://www.hindawi.com
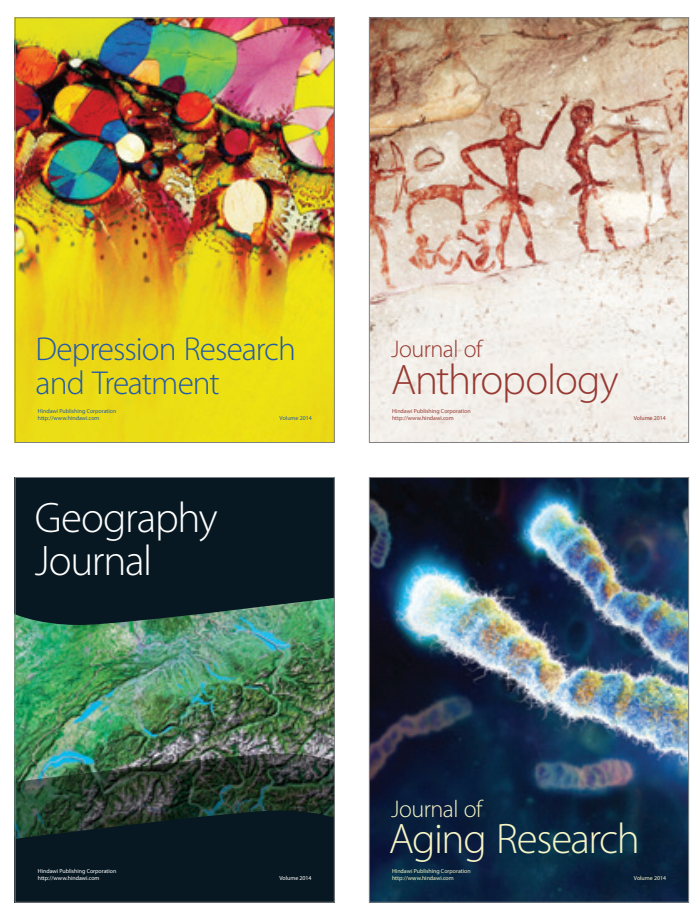

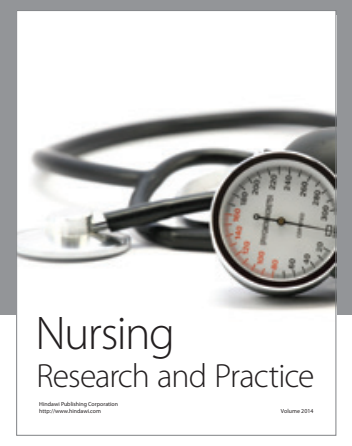

Nursing

Research and Practice

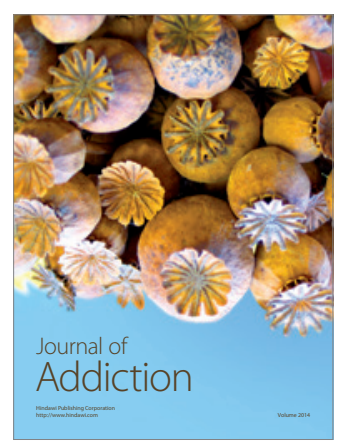

Child Development

Research

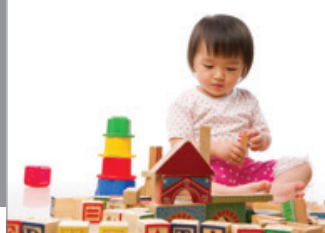

迥
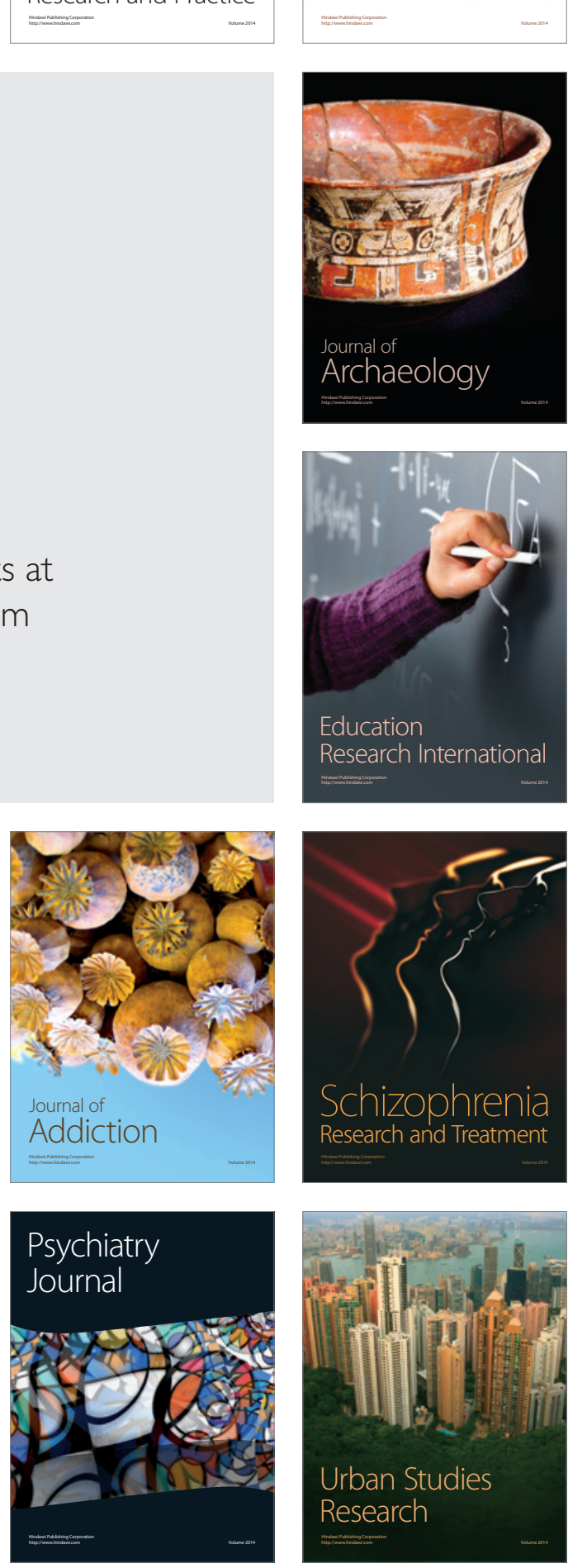